Recepción: 30 / 10 / 2017

Aceptación: 25 / $11 / 2017$

Publicación: 15/ 12/ 2017

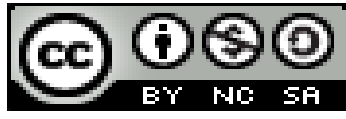

Ciencias económicas y empresariales

Artículo de investigación

\title{
Optimización de potencia en transformadores de distribución
}

Power optimization in distribution transformers

\section{Otimização de energia em transformadores de distribuição}

\author{
Jaime J. Báez-Zamora ${ }^{\mathrm{I}}$ \\ jesusjaimebaez@hotmail.com \\ Byron F. Chere-Quiñónez II \\ cherokyfernando@hotmail.com \\ Alejandro J. Martínez-Peralta III \\ pipoperalta1990@hotmail.com \\ Karen Y. Simisterra-Quiñónez ${ }^{\text {IV }}$ \\ krenyane@gmail.com \\ Freddy F. Portocarrero-Rivas V \\ f_porto_27@hotmail.com
}

Correspondencia: jesusjaimebaez@hotmail.com

I. Ingeniero Eléctrico, Docente Universidad Técnica Luis Vargas Torres de Esmeraldas, Esmeraldas, Ecuador.

II. Ingeniero Eléctrico, Docente Universidad Técnica Luis Vargas Torres de Esmeraldas, Esmeraldas, Ecuador.

III. Ingeniero Eléctrico, Docente Universidad Técnica Luis Vargas Torres de Esmeraldas, Esmeraldas, Ecuador.

IV. Magíster en Administración de Empresas Mención Planeación, Ingeniero Eléctrico, Docente Universidad Técnica Luis Vargas Torres de Esmeraldas, Esmeraldas, Ecuador.

v. Ingeniero Eléctrico, Docente Universidad Técnica Luis Vargas Torres de Esmeraldas, Esmeraldas, Ecuador. 


\section{Resumen}

El presente estudio trata de la optimización de potencia en un parque de transformadores de un sistema de distribución, en el cual se implementó y aplico un método grafico para la estimación y optimización de la potencia en transformadores. Este método se complementó con el utilizado convencionalmente en las empresas de distribución de energía, brindando así mayor claridad al momento de analizar el comportamiento de los elementos que conforman la fusión de la demanda eléctrica, cuyos elementos principales son los usuarios, el tiempo y la energía, la cual varia con respecto a estas dos variables antes mencionadas. Por otro lado, también se trata del desarrollo de una aplicación en un software convencional como lo es Excel y su herramienta VBA visual Basic ya que este software brinda una variedad de aplicaciones de fácil uso y desarrollo, junto con el software Matlab. Esta aplicación combina dos métodos los cuales se complementan entre sí, facilitando los cálculos de la demanda y explicando didácticamente cómo se comportan las demandas máximas, coincidentes y no coincidentes. A esto se le agregó una función optimización basada en el estudio de los alimentadores de la cuidad con mayor carga residencial, los cuales representan el comportamiento de los usuarios residenciales de la zona que tienen una curva de demanda diaria característica cuyos factores de variación horaria sirvieron para optimizar la curva de demanda diaria de los trasformadores de distribución.

Palabras clave: optimización; transformadores; métodos; Excel VBA; Matlab.

\section{Abstract}

The present study deals with the optimization of power in a transformer park of a distribution system, in which a graphical method for estimating and optimizing the power in transformers was implemented and applied. This method was complemented with the conventionally used in energy distribution companies, thus providing greater clarity when analyzing the behavior of the elements that make up the merger of electricity demand, whose main elements are users, time and energy, which varies with respect to these two variables mentioned above. On the other hand, it is also about the development of an application in a conventional software such as Excel and its visual VBA Basic tool since this software provides a variety of applications of easy use and development, together with the Matlab software. This application combines two methods which complement each other, facilitating the calculation of the demand and explaining didactically 
how the maximum, coinciding and non-coinciding demands behave. To this was added an optimization function based on the study of city feeders with higher residential load, which represent the behavior of residential users in the area who have a characteristic daily demand curve whose time variation factors served to optimize the daily demand curve of the distribution transformers.

Keywords: optimization; transformers; methods; Excel VBA; Matlab.

\section{Resumo}

O presente estudo trata da otimização do poder em um parque de transformadores de um sistema de distribuição, no qual foi implementado e aplicado um método gráfico para estimar e otimizar a potência nos transformadores. Este método foi complementado com as convencionalmente utilizadas em empresas de distribuição de energia, proporcionando maior clareza ao analisar o comportamento dos elementos que compõem a incorporação da demanda de eletricidade, cujos elementos principais são usuários, tempo e energia, que varia em relação a estas duas variáveis mencionadas acima. Por outro lado, é também sobre o desenvolvimento de uma aplicação em um software convencional, como o Excel e sua ferramenta Visual VBA Basic, uma vez que este software fornece uma variedade de aplicações de fácil utilização e desenvolvimento, juntamente com o software Matlab. Esta aplicação combina dois métodos que se complementam, facilitando o cálculo da demanda e explicando de forma didática como as demandas máximas, coincidentes e não coincidentes se comportam. Para isso foi adicionada uma função de otimização baseada no estudo de alimentadores de cidades com maior carga residencial, que representam o comportamento de usuários residenciais na área que possuem uma curva de demanda diária característica cujos fatores de variação de tempo serviram para otimize a curva de demanda diária dos transformadores de distribuição.

Palavras chave: otimização; transformadores; métodos; Excel VBA; Matlab.

\section{Introducción}

El análisis del comportamiento de la demanda actual de energía eléctrica es muy importante, ya que es un factor del cual depende directamente el sostenimiento y desarrollo de los sectores residencial, industrial y comercial en el país. 
Los usuarios residenciales están en constante crecimiento por lo que es necesario un correcto dimensionamiento del sistema de distribución en el que están o estarán conectados, con la finalidad de proveerles una energía continua y de la mayor calidad posible. Para ello algunas de las empresas distribuidoras de energía eléctrica del país, han construido curvas de demanda máxima diaria (CONELEC, s/a), que modelan el comportamiento de los usuarios del sistema eléctrico de distribución de su respectiva área de concesión, sin embargo, existe todavía incertidumbre al momento de dimensionar redes para media y baja tensión ya que las empresas eléctricas de algunas ciudades no cuentan con un estudio en el que se pueda basar el diseñador para dimensionar correctamente las redes de distribución y así evitar el sobre dimensionamiento de la red o el sub direccionamiento de la misma.

Para una apropiada estimación de potencia no hay que basar los criterios o en estudios realizados fuera del área concesión de la empresa eléctrica donde se ejecutara el proyecto, en apreciaciones subjetivas y en argumentos con demasiada incertidumbre, por tal razón es necesario conocer la naturaleza de las cargas que van a estar conectadas al alimentador y el comportamiento de los usuarios que se van a servir de los diferentes equipos eléctricos que estarán conectados a los trasformadores de distribución. Por otro lado, las costumbres de los usuarios de equipos eléctricos, no pueden ser generalizadas en el país, ya que ella depende de diversos factores, cuyas cualidades cuantitativas y cualitativas, están directamente relacionados con su ubicación geográfica y el nivel socio-económico. Por esto es necesario implementar un método de estimación de la demanda de potencia que utilice la ubicación geográfica de los usuarios y él nivel socio económico para generar curvas de demanda que modele el comportamiento de los usuarios de la red.

En el país, existen varios métodos que son utilizados para determinar la demanda de potencia a nivel macro y microeconómico, algunos de ellos están basados en la curva de demanda máxima coincidente de la empresa eléctrica Quito y otros en apreciaciones de cada diseñador, la EEQ posee tablas que son resultado de un estudio realizado en el área de concesión de dicha empresa, la cual no tiene total correspondencia con las costumbres en el uso de equipos eléctricos de los usuarios de otra área del país. Por tal motivo la curva de carga diaria de los equipos eléctricos de una vivienda ubicada en la cuidad de Esmeraldas, no tienen correspondencia con la curva de

\section{4}

Pol. Con. (Edición núm. 14) Vol. 2, No 12, diciembre 2017, pp. 31-43, ISSN: 2550 - 682X 
carga de los equipos de una vivienda que está ubicada en la ciudad de Quito; por motivos de la altitud y temperatura ambiente promedio, las cuales modifican las costumbres de los consumidores de energía eléctrica, que se encuentran en dichas ciudades en sus diferentes estratos de consumo. Para no sobre dimensionar la red de distribución y también no sub dimensionarla, se ha implementado el método Arvidson optimizado para el cálculo de la demanda de los transformadores de distribución, el cual nos da un mayor control de las pérdidas de potencia, y nos ayuda a crear factores de coincidencia y diversidad basándose en el estudio de carga propio de cada proyecto de distribución de energía, lo cual economiza la inversión inicial y posterior de la red eléctrica atreves de la optimización de la potencia de los transformadores de distribución que se usen en cada proyecto.

Este trabajo tiene como objetivo general: Presentar una herramienta de estimación de potencia con vistas a optimizar la potencia de transformadores de distribución aplicando factores de variación horaria obtenidos de la curva de demanda diaria local.

\section{Métodos}

Las características de carga pueden determinarse a partir de mediciones de campo, o mediante análisis estadísticos. Para los sistemas eléctricos existentes, las características de carga pueden obtenerse a partir de mediciones; no así para los sistemas nuevos, los mismos que se requiere compararlos con los existentes, de los cuales se pueden obtener datos y mediciones precisos.

Los datos que son necesarios, para obtener las características de carga se pueden tener a partir de sus cualidades básicas las cuales son: la demanda máxima y la demanda promedio misma que significa el número de KWH consumidos, en un intervalo de tiempo definido, sobre el número de horas equivalentes a ese ese lapso de tiempo.

Para determinar las características en grupos de cargas no similares se procede a aislar la carga de interés de las otras, resultando este proceso muy caro por el monto económico que genera realizar las mediciones, el cual puede superar el valor de los ingresos de una empresa eléctrica. 
Se realiza el análisis probabilístico a partir de experiencias en redes de distribución europeas, en las que se han obtenido mediciones dan a entender que la variación de carga en una red de distribución puede considerarse, por lo general, en forma bastante aproximada, como de distribución estadística normal durante los altos períodos de carga (Orejuela, 1980).

Los métodos gráficos en base al promedio de demanda máxima diversificada por consumidor se definirán como A y B:

El método A considera la diversidad entre cargas similares, como también la no coincidencia de los picos, de los distintos tipos de carga. Como última consideración, se incluye al 'factor de variación horaria', que no es más que la relación entre la demanda a una determinada hora, de un tipo particular de carga, y la demanda máxima de aquel tipo particular de carga (Castaño, 2006). Este método utiliza una familia de curvas.

El método B hace uso de curvas, las cuales representan las características del promedio de la demanda máxima por cada usuario consumido, para varios tipos de electrodomésticos. Este método no toma en cuenta los factores de variación horaria.

El método en base a la relación entre demanda máxima y kwh consumidos elimina la dificultad que se genera al realizar las mediciones de la demanda máxima, porque considera el cálculo de los KWH, consumidos por mes y por abonado, valor que se obtiene con facilidad de las planillas facturadas por las empresas eléctricas. Hay dos formas distintas para determinar la demanda máxima diversificada de un grupo de $\mathrm{n}$ número de usuarios y también usando como datos el número de KWH de consumo, el número de clientes del servicio eléctrico.

Para este trabajo se aplica el método ARVIDSON, el cual utiliza tiempo y la cantidad de usuarios como variables para el cálculo de la demanda máxima coincidente. Se usan trasformadores de la urbanización Alta Monte que se encuentra ubicada en la vía TACHINA - TIGRE, parroquia Tachina, cantón y provincia de Esmeraldas.

Las demandas máximas coincidentes y no coincidentes, son la base para el cálculo de las potencias que dimensionarán los transformadores de distribución que son elementos claves para optimizar la potencia de una red de distribución. 
Los factores climáticos influyen en el uso de algunos equipos eléctricos, en el caso de la Urbanización, se presenta un clima cálido por lo que se les dará mucho énfasis a los aires acondicionados y ligeramente se aumentará la potencia de las cocinas de inducción con respecto a las cocinas de la región Sierra del Ecuador.

La aplicación que se desarrolla combina dos métodos, el de las demandadas coincidentes respecto de los usuarios y el de la demandada coincidente respecto de los usuarios y el tiempo.

La aplicación de Visual Basic posee un In Put, en el cual van los datos de entrada, como lo son el número de usuarios, nivel socioeconómico, taza de crecimiento de la demanda, tipo de transformador etc. En el Out Put van los datos de salida en donde se detallan las variables que modelan el comportamiento de la demanda en los cuales se encuentra el factor de diversidad, la demanda máxima diversificada, las pérdidas de potencia, el factor de carga y de demanda. Los resultados dan factores de diversidad correspondientes a la curva de carga que se ingresó al programa como base de datos normalizando previamente ajustados en Matlab con su herramienta cftool (MathWorks, 2016).

Para la aplicación de este método se ha tomado como ejemplo el sector \# 10 de la urbanización pues este es el sector que más usuarios tiene.

La aplicación de Excel y VBA (visual Basic) necesitó el ajuste de las curvas de demanda promedio de cada equipo eléctrico ya que no se encontraron tablas que grafiquen una curva uniforme, con la que se pueda manejar una ecuación que modele el comportamiento de la potencia con respecto de los usuarios. Este ajuste se lo realizó con el software Matlab obteniendo los factores A, B, C que sirven para modelar la curva de carga de potencia vs número de usuarios.

El valor de la demanda de más de 100 usuarios, se obtuvo a partir de las contantes obtenidas en el software Matlab mismas que fueron ingresadas en Excel para obtener las tablas de demanda promedio coincidente.

Para la aplicación del método Arvidson optimizado se ha tomado como ejemplo el transformador CT-10 de 50 KVA perteneciente al parque de transformadores de la urbanización Alta Monte. El sector que se escogió para el estudio es el que tiene más usuarios. 
El transformador de este sector sirve a 22 usuarios tipo B, según las normas de la EEQ, perteneciente al parque de transformadores de la red primaria de la urbanización. La aplicación desarrollada en Excel VBA utiliza del método Arvidson para la optimización de potencias, el cual toma en cuenta la no coincidencia de los picos de las demandas y las coincidencias para calcular la demanda máxima diversificada.

La potencia de algunos de los transformadores se optimizó por medio de la curva de demanda diaria de la zona donde está ubicado el transformador, esto se hace para optimizar los picos de demanda en cada hora del día, si existe una demanda a determinada hora del día, que no coincida con la curva de demanda diaria de la zona, esta se reducirá o aumentará; si esta demanda se encuentra en la hora pico de la curva de demanda diaria que caracteriza la zona, esta demanda no se modificará.

Para optimizar se procedió a hacer mediciones en los principales alimentadores de la cuidad con carga residencial, los cuales representan muy bien a los usuarios residenciales de la cuidad de Esmeraldas. Los alimentadores en los cuales se hizo las mediciones son: El alimentador Balao (sector de nuevos horizontes y santa Cruz), Tolita (Tilita 1y Tolita 2), Sub Chico (Santas Barrio Lindo), San Rafael (50 Casas voluntad de Dios), Pradera (Altos de Jardines de la paz) y Malecón Centro (Malecón y Centro).

La muestra de las mediciones fue tomada en intervalos de 15 minutos, durante las 24 horas del día, en cada alimentador en los meses de mayor demanda (agosto y diciembre). En los cuales, previo análisis, se tomaron tres alimentadores para sacar las muestras de los días con sus respectivas demandas horarias, los cuales fueron el alimentador Tolita, Malecón centro y el alimentador balao.

La demanda máxima diversificada (DMD) se calcula a partir de la carga instalada de una casa del nivel socio económico $\mathrm{B}$, para esto se ha usado la encuesta de INEC donde asume que un usuario tipo B es el segundo estrato y representa el 11,2\% de la población investigada.

En cuanto a las viviendas: 
- En el 46\% de los hogares, el material predominante del piso de la vivienda es de duela, parquet, tablón o piso flotante.

- En promedio tienen dos cuartos de baño con ducha de uso exclusivo para el hogar.

- El 97\% de los hogares dispone de servicio de teléfono convencional.

- E1 99\% de los hogares cuenta con refrigeradora.

- Más del 80\% de los hogares dispone de cocina con horno, lavadora, equipo de sonido y/o mini componente.

- En promedio los hogares tienen dos televisiones a color.

La potencia de los equipos eléctricos se toma de la placa de cada equipo de una vivienda, luego se la ubica en una categoría y finalmente se suman todos los equipos de aquella categoría. Existen también tablas normalizadas que se pueden tomar como referencia de potencia de cada electrodoméstico.

Una vez obtenidos estos datos se procede a ingresar las cargas de la vivienda presionando el botón correspondiente a cada electrodoméstico, los botones que quedan macados representan los equipos que van a estar funcionando en la vivienda. Con esto se define el nivel socio económico, ya este está en función de los electrodomésticos que tiene cada usuario. El porcentaje de la curva de cada electrodoméstico que se va a usar, lo define los números de color amarillo y fondo negro, en el panel de selección de equipos eléctricos. Se debe activar el botón DMDcoin/no-coin y ubicar el número de usuarios en 1 para hallar la carga instalada y la demanda máxima unitaria de la casa modelo.

Luego se presiona el botón line para ingresar la carga instalada la cual representa el consumo que tengan los electrodomésticos en $\mathrm{kw} / \mathrm{h}$. Luego se ingresa el FFU (\%). Una vez hecho esto se obtiene las curvas de demanda promedio de los diferentes electrodomésticos

Para no sobredimensionar la demanda de las cocinas de inducción y del aire acondicionado se procede a calcular el promedio entre el porcentaje de FFU (EEQ, 2014) y el porcentaje proyectado de los usuarios que poseen la cocina de inducción y el aire acondicionado respectivamente. En este caso la curva de la demanda diaria es lineal, porque no se han aplicado todavía los factores de variación horaria, que se procede hacerlo a continuación. 
Después de este paso se desactiva el botón line y se obtienen los ciclos de la demanda diaria del transformador, donde existe una interacción entre la demanda de potencia con respecto al número de usuarios y el tiempo.

Luego se obtiene la demanda máxima unitaria y diversificada para el número de usuarios ingresados, en el caso del ejemplo tenemos 22 usuarios residenciales tipo B.

El cálculo de la demanda máxima diversificada o coincidente depende de la cantidad de usuarios y es con la que se calculan los factores de coincidencia y factores de diversidad. El botón OPTM está activo, por lo que la demanda máxima coincidente esta optimizada. La demanda máxima coincidente la tuvo la iluminación y todas las misceláneas a las 20:00 horas.

Al obtener estos datos se procede a ingresar o calcular las cargas-especiales y alumbrado-público. Luego de esto se proyecta la demanda a 10 años, con un Ti (taza de crecimiento para usuarios tipo B) de $2.5 \%$ anual (EEQ, 2014).

A continuación, se procede a hallar las pérdidas de potencia en función del factor de carga (Castaño, 2006).

Luego se escoge el transformador de distribución adecuado para la potencia calculada que va servir a los usuarios, en este caso uno con una cargabilidad de $98.23 \%$ lo cual es soportable para este tipo de transformadores, ya que no es afectado su rendimiento debido a que los transformadores que sirven a un estrato socio económico B pueden soportar una sobrecarga del $10 \%$ de su potencia nominal. Y este nivel de cargabilidad será alcanzado aproximadamente en los siguientes 10 años.

Para tener mayor confianza en que el transformador rendirá adecuadamente se deberá hacer las respectivas pruebas de rendimiento y cortocircuito que corresponden a los transformadores de distribución.

Finalmente se presiona el botón analizar el cual hace que se muestran los datos de salida de la aplicación correspondientes a la demanda del transformador 


\section{Desarrollo}

La demanda la Urbanización Alta Monte es de 412.5 KVA con el método tradicional; según el método Arvidson. La potencia que requerirá la urbanización es de $400 \mathrm{KVA}$. El método de estimación de potencia de la EEQ se hace con las tablas de demanda coincidentes factores $\mathrm{F}$ y N. Para dimensionar las pérdidas se basó en las siguientes fórmulas.

Con un factor de carga para un intervalo de 25 min., $\%$ Per $=0.3 F c+0.7 F c^{2}$ y $D p e r=\%$ Per $x D M D$. La potencia requerida es $393.82 \mathrm{KVA}$.

La optimización de potencia se debe realizar en usuarios que no estén delimitados como urbanización, ya que en este tipo de proyectos la DMU es fija y estos son minoría. En la participación que tienen en la curva de demanda diaria de la zona, estos deben pertenecer a zonas urbanizadas no restringidas. En nuestro caso se estudia el nivel socio económico C + (según lo estipulado en el censo del INEN) que es el equivalente del tipo B de la urbanización estudiada.

Cuando se trata de optimizar un recurso es necesario analizar parámetros que sean elementos de la función que genera la demanda, en el caso que se estudia, dichos elementos son el nivel socio económico de la zona del transformador, la tasa de crecimiento de la población en ese sector, el índice de utilización del suelo y los horarios de la demanda de energía en la zona. Nos centraremos en este último, ya que optimizaremos la potencia considerando la variable tiempo pues existen zonas que tienen una curva de demanda diaria característica, la cual se puede utilizar para delimitar o restringir varios picos de demanda horaria, optimizando así los picos de potencia que están por encima o por debajo de la curva característica de la zona del proyecto.

La curva de demanda diaria de la zona fue resultado de mediciones hechas en un intervalo de 15 minutos en los alimentadores con mayor carga residencial de Esmeraldas. La potencia requerida optimizada es $295.38 \mathrm{KV}$.

Los precios fueron sacados de la lista de precios en transformadores ABB (ABB, 2016). 
Los trasformadores con carga diferente a la carga residencial se excluyeron del cálculo por lo que sus potencias no varían.

\section{Conclusiones}

Se optimizó la potencia de un parque de transformadores cuya demanda de potencia normalizada es de 412.5 KVA, haciendo el respectivo estudio de la curva de carga diaria de la zona y multiplicando la demanda calculada en cada hora del día, por los factores de variación horaria obtenidos del estudio de optimización propuesto, se llegó a una potencia de $362.5 \mathrm{KVA}$ los cuales son válidos en áreas con una DMU (Demanda máxima unitaria) promedio con un índice de variación moderado. La aplicación de este método de optimización fue hecha con base en estudios ya existentes, (En el plan maestro de electrificación 2013-2022, Normas EEQ, cálculo de demandas de diseño) en las Normas IEC.

Se desarrolló una herramienta de estimación de potencia por medio de un Software convencional como lo es Excel y su herramienta Visual Basic. También se usó el software Matlab el cual sirvió para el ajuste de la curva de datos de la demanda promedio coincidente de equipos eléctricos Para luego ser ingresados como base de datos al software Excel.

También se determinaron los factores de coincidencia aplicables a usuarios residenciales, los cuales se pueden obtener de la curva de demanda promedio coincidente de cada empresa de distribución. La aplicación que se desarrolló en Excel VBA da la libertad de cambiar la curva de la demanda promedio coincidente y así cambiar los factores de coincidencia y diversidad.

\section{Referencias Bibliográficas}

ABB. (2016). Lista de precios No PTM PL 001 ES. Transformadores de distribución monofásicos y trifásicos tipo poste $\mathrm{o}$.

Castaño, S. (2006). Redes de Distribución de Energía. Colombia: Universidad Nacional de Colombia.

CONELEC. (s/a). Estudio y gestión de la demanda eléctrica. Plan maestro de electrificación. 
EEQ. (2014). Normas para Distribución. Quito.

INEC. (2017). http://www.ecuadorencifras.gob.ec/encuesta-de-estratificacion-del-nivelsocioeconomico/.

MathWorks. (2016). Curve Fitting app. MATLAB® Tools.

Orejuela, V. (1980). Comparación de demandas de diseño en sistemas eléctricos de distribución. Quito.

Torres, R. (2006). Tecnología Eléctrica. Madrid: Mc Graw Hill.

Vicuña, C. G. (2017). Electro Industria. 